\title{
Modulating internal transition kinetics in responsive macromolecules by collective crowding
}

\author{
Upayan Baul, ${ }^{1}$ Nils Göth, ${ }^{1}$ Michael Bley, ${ }^{1}$ and Joachim Dzubiella ${ }^{1,2}$ \\ ${ }^{1}$ Applied Theoretical Physics - Computational Physics, Physikalisches Institut, \\ Albert-Ludwigs-Universität Freiburg, D-79104 Freiburg, Germany \\ ${ }^{2}$ Cluster of Excellence livMatS@FIT-Freiburg Center \\ for Interactive Materials and Bioinspired Technologies, \\ Albert-Ludwigs-Universität Freiburg, D-79110 Freiburg, Germany
}

(Dated: September 24, 2021)

\begin{abstract}
Packing and crowding are used in biology as mechanisms to (self-)regulate internal molecular or cellular processes based on collective signalling. Here, we study how the transition kinetics of an internal 'switch' of responsive macromolecules is modified collectively by their spatial packing. We employ Brownian dynamics simulations of a model of 'responsive colloids' (RCs), in which an explicit internal degree of freedom - here, the particle size - moving in a bimodal energy landscape responds self-consistently to the density fluctuations of the crowded environment. We demonstrate that populations and transition times for the two-state switching kinetics can be tuned over one order of magnitude by 'self-crowding'. An exponential scaling law derived from a combination of Kramers' and liquid state perturbation theory is in very good agreement with the simulations.
\end{abstract}


Over the last two decades the phenomenon of macromolecular crowding has attracted great attention because of its large effects on biomolecular mobility, reactions, structure and dynamics [1 5]. In particular, crowding leads to variations of (two-state) folding energy landscapes and kinetics, typically leading to more compact molecular states and speeding up folding times [6 9]. In fact, it was proposed that cells can regulate and maintain a relatively constant level of packing of macromolecules to tune molecular processes, which was termed 'homeocrowding' as an acronym of macromolecular crowding homeostasis [10].

Similar mechanisms play decisive roles also on larger scales, for example, in bacterial 'quorum sensing', which is the self-regulation of internal gene expression rates in response to fluctuations in overall cell-population density [11]. These fascinating collective effects are inspiring for the design of 'intelligent' synthetic materials, where soft and responsive particles, e.g., polymer-based hydrogels, potentially mimic these effects for discovering new functionalities, such as advanced time responses, feedback and adaptivity [12, 13]. In particular, the colloid/polymer duality of soft microgels [14, 15] and their stimuli-triggered conformational switching [16] open new avenues for creating programmable, self-regulating materials.

Despite the progress in this field, our understanding is still based on relatively simple soft matter concepts such as excluded volume, depletion interactions, or scaled particle theory [10, 17]. In other words, in conventional theoretical studies of macromolecular crowding the crowders are hard and inert, while in reality they are soft and responsive. Like in bacteria, control by crowding is collective and self-consistent, affecting the crowder and the crowded simultaneously [11]. Physical models that self-consistently address crowding effects and their consequence on internal processes, such as internal transitions or reactions, are still absent. A promising approach in this regard is the explicit consideration of internal degrees of freedom (DOFs) which can respond to environmental changes [18 23], including local density fluctuations [24].

In this letter, we study collective crowding effects for a single macromolecular species ('self-crowding') employing a model of Responsive Colloids (RCs) [19, 24]. The latter explicitly resolves an internal 'property' DOF that responds self-consistently to changes in the local packing. As a case study, we focus on the two-state switching kinetics of the size of the macromolecule, modeling, for example, conformational transitions in globular protein folding [6 8], switching of intrinsically disordered proteins (IDPs) [25] or synthetic supramolecular polymers [26], or bimodal hydrogel volume transitions [12, 27 29]. We find 
that populations and the transition times between the two sizes (small and large) modified by packing density spread over one order of magnitude. Importantly, we develop a scaling theory based on a combination of Kramers' escape [30] and liquid state perturbation theory [31] extended to RCs. The theory is in very good agreement with the calculated kinetics and provides useful, experimentally testable scaling laws for a general class of Hamiltonians.

We consider $N$ interacting particles in a volume $V$ with number density $\rho=N / V$ in absence of any external fields. The coarse-grained RC Hamiltonian is defined as [19]

$$
H\left(\mathbf{r}^{N}, \sigma^{N}\right)=\sum_{i}^{N} \psi\left(\sigma_{i}\right)+\frac{1}{2} \sum_{i \neq j}^{N} \phi\left(\mathbf{r}_{i}, \mathbf{r}_{j} ; \sigma_{i}, \sigma_{j}\right),
$$

and describes the energy of the microstate of the configuration $\left\{\mathbf{r}_{i}, \sigma_{i}\right\}=:\left\{\mathbf{x}_{i}\right\}$, where the $\mathbf{r}_{i}$ and $\sigma_{i}$ are positions and sizes of particle $i=1 . . N$, respectively. The first term on the right-hand-side carries the one-body contribution to the energy associated with changes in the size $\sigma$. Thus, it represents the energy landscape $\beta \psi(\sigma)=-\ln p(\sigma)$, where $p(\sigma)$ is the corresponding probability distribution, and $\beta=1 / k_{B} T$ the inverse thermal energy. We refer to $p(\sigma)$ as the parent distribution since it describes the intrinsic internal polydispersity of an isolated particle. To model two-state kinetics, we choose a bimodal form of the explicit, double-Gaussian form

$$
p(\sigma)=\frac{1}{\sqrt{2 \pi}}\left[\frac{c}{\delta_{1}} e^{\left(-\frac{1}{2}\left(\frac{\sigma-\mu_{1}}{\delta_{1}}\right)^{2}\right)}+\frac{1-c}{\delta_{2}} e^{\left(-\frac{1}{2}\left(\frac{\sigma-\mu_{2}}{\delta_{2}}\right)^{2}\right)}\right] .
$$

This and its corresponding energy landscape $\psi(\sigma)$ are shown in Fig. 1(a), highlighting the energy minima $\mu_{1}, \mu_{2}$, and the local maximum, defining the transition state (TS). In going from continuous distributions to the two-state (small (S) versus large (L)) picture, we consistently use the TS as the state boundary. In our study, $c=0.5, \mu_{1}=0.63 \sigma_{0}, \mu_{2}=1.0 \sigma_{0}$, and $\delta_{1}=\delta_{2} \equiv \delta=0.1 \sigma_{0}$, with unit length $\sigma_{0}$ set to 1.0. The choice leads to an unperturbed activation energy barrier $E_{0}^{a} \simeq 1 k_{B} T$ (Fig. 1(a)).

The second term in Eq. (1) represents the two-body, effective pair potential, $\phi\left(\mathbf{r}_{i}, \mathbf{r}_{j} ; \sigma_{i}, \sigma_{j}\right)$, which is explicitly dependent on both the positions and the property of the interacting RCs. Here, we choose the Hertzian potential

$$
\phi\left(r ; \sigma_{i}, \sigma_{j}\right)=\epsilon\left(1-\frac{r}{\sigma_{i j}}\right)^{5 / 2} \Theta\left(1-\frac{r}{\sigma_{i j}}\right),
$$

where $\Theta$ is the Heaviside step function, $\epsilon$ dictates the magnitude of the interaction, $r=$ $\left|\mathbf{r}_{j}-\mathbf{r}_{i}\right|$ is the interparticle distance, and $\sigma_{i j}=\left(\sigma_{i}+\sigma_{j}\right) / 2$. The Hertzian potential reflects 
the soft, purely repulsive interactions between spherical elastic colloids with diameter $\sigma_{i}$, widely used to describe swollen microgels [32 35. For our RC system we choose $\beta \epsilon=500$, which is in the typical range for modeling real experimental systems [34].

The equilibrium probability distribution of sizes in the dispersion is defined as $N(\sigma ; \rho)=$ $\frac{1}{N}\left\langle\sum_{i=1}^{N} \delta\left(\sigma-\sigma_{i}\right)\right\rangle$ by the canonical ensemble average. It represents the emergent property distribution accounting for the effects of interactions among RCs. It is a central in our study, because

$$
\beta \tilde{\psi}(\sigma ; \rho)=-\ln N(\sigma ; \rho)
$$

defines the crowding-modified energy landscape, which tunes the internal two-state switching. Fig. 1(b) exemplifies the modified energy barrier $E_{\mathrm{SL}}^{a}$ for $\mathrm{S}$ to $\mathrm{L}$ transitions. Since we have a Hamilton system with explicit response of the sizes, the modification of the energy landscape is collective and self-consistent.

We assume both the translation and the size dynamics to be overdamped and propagated by Brownian dynamics (BD) [36], as

$$
\mathbf{x}_{i}(t+\Delta t)=\mathbf{x}_{i}(t)+\frac{D_{l, i}}{k_{B} T} \mathbf{F}_{i}(t) \Delta t+\boldsymbol{\xi}_{i}
$$

where $\Delta t$ is the simulation timestep. $\quad \mathbf{F}_{i}=-\nabla_{i} H$ is the generalized force experienced by $i$-th particle from pairwise interactions given Hamiltonian $H$, Eq. (1), and $\nabla=$ $(\partial / \partial x, \partial / \partial y, \partial / \partial z, \partial / \partial \sigma)$ is a 4 -gradient. The term $\boldsymbol{\xi}_{i}$ represents stochastic forces stemming from the solvent fluctuations. For the evolution along a coordinate $l=x, y, z, \sigma$, the corresponding component $\xi_{l}$ is drawn from a Gaussian distribution with mean $\left\langle\xi_{l}\right\rangle=0$ and variance $\left\langle\xi_{l}^{2}\right\rangle=2 D_{l} \Delta t$, which is strictly $\delta$-correlated in time and obeys the standard fluctuation-dissipation theorem [37]. For translation $(l=x, y, z)$, the diffusivity $D_{l, i}$ in Eq. (5) is defined in units $\sigma_{0}^{2} / \tau_{\mathrm{BD}}$, where $\tau_{\mathrm{BD}}$ sets our unit (Brownian) time scale. The diffusion is related to the friction coefficient $\zeta_{l, i}$ through $D_{l, i}=k_{B} T / \zeta_{l, i}$. We assume the latter to follow the Stokes-Einstein relation $\zeta_{l, i} \propto \sigma_{i}$, as recently verified for conformationally fluctuating proteins [38]. Setting the time scale of size $(\sigma)$ fluctuations, we define $D_{\sigma}=\alpha \sigma_{0}^{2} / \tau_{\mathrm{BD}}$ and use the parameter $\alpha$ (same for all particles) to tune the intrinsic time scale of internal switching.

We simulate fully periodic systems in the canonical ( $N V T$ ) ensemble comprising $N=512$ RCs for $\alpha=(0.001,0.01,0.1,1.0,10.0)$ and particle densities in the range from the low density limit (LDL), $\rho \sigma_{0}^{3} \rightarrow 0$, up to $\rho \sigma_{0}^{3}=1.91$. The LDL is approximated by simulating 
(a)

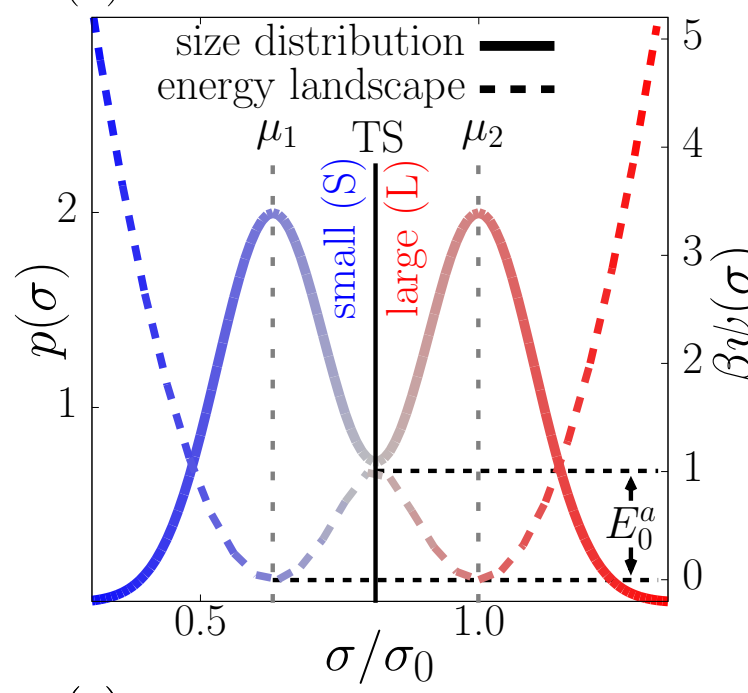

(c)

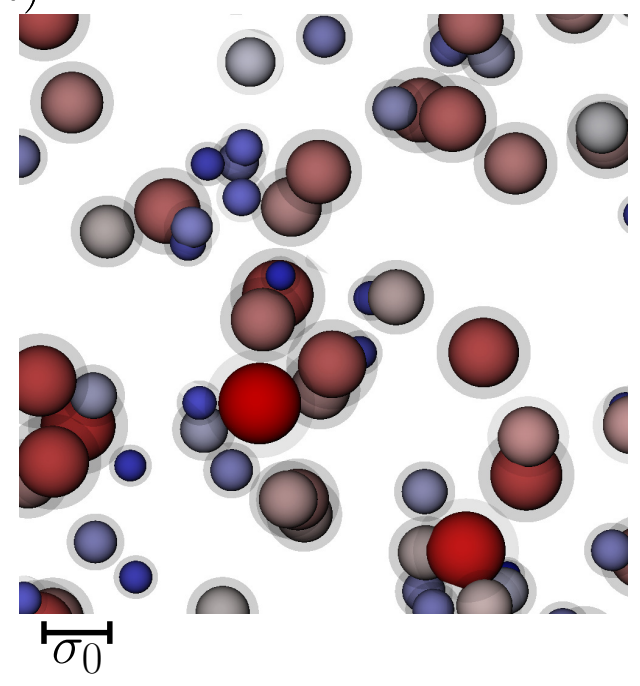

(b)

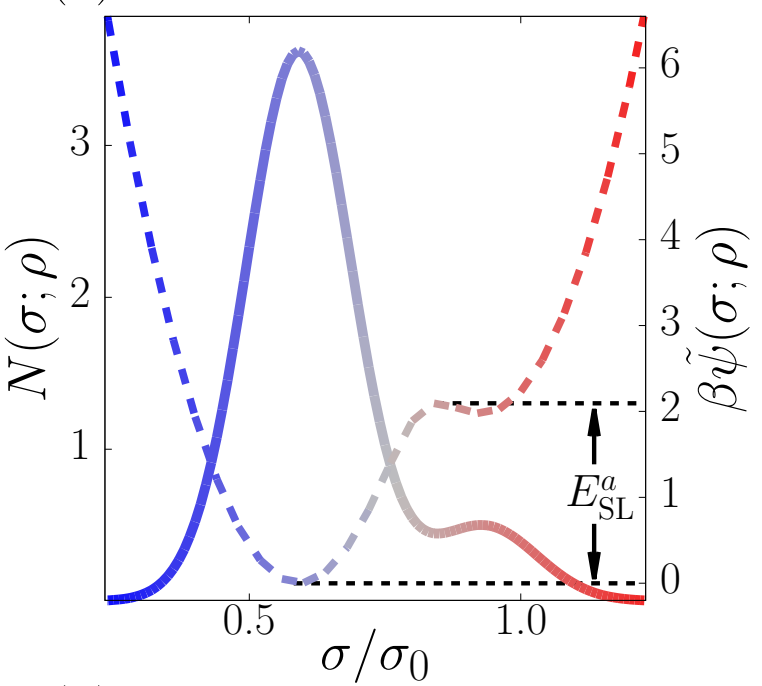

(d)

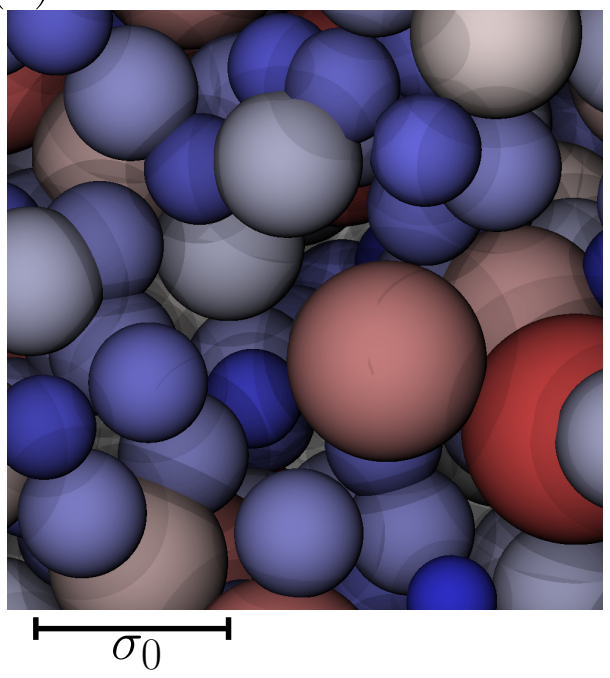

FIG. 1. (a) Parent distribution $p(\sigma)$ of size $\sigma$ (solid) and corresponding energy landscape $\psi(\sigma)$ (dashed) at dilute (single-particle) conditions. Locations of energy minima $\left(\mu_{1}, \mu_{2}\right)$ and the corresponding transition state (TS) are shown, separating small (S, blue) and large (L, red) states. (b) Example emergent distribution $N(\sigma ; \rho)$ (solid) and corresponding energy landscape $\tilde{\psi}(\sigma ; \rho)$ (dashed). The crowding changes the activation energy from $E_{0}^{a}$ in (a) to $E_{\mathrm{SL}}^{a}$ in (b). (c, d) Representative snapshots of system configurations under dilute and crowded conditions, respectively. The color gradient from blue (small sizes) to red (large sizes) visualizes the dynamical sizes according to the distributions in the top panels (note the scale bars).

an isolated particle. More simulation details are given elsewhere [39]. Simulation snapshots with particle size distributions resolved by color are illustrated in Figs. 1(c) and (d). 

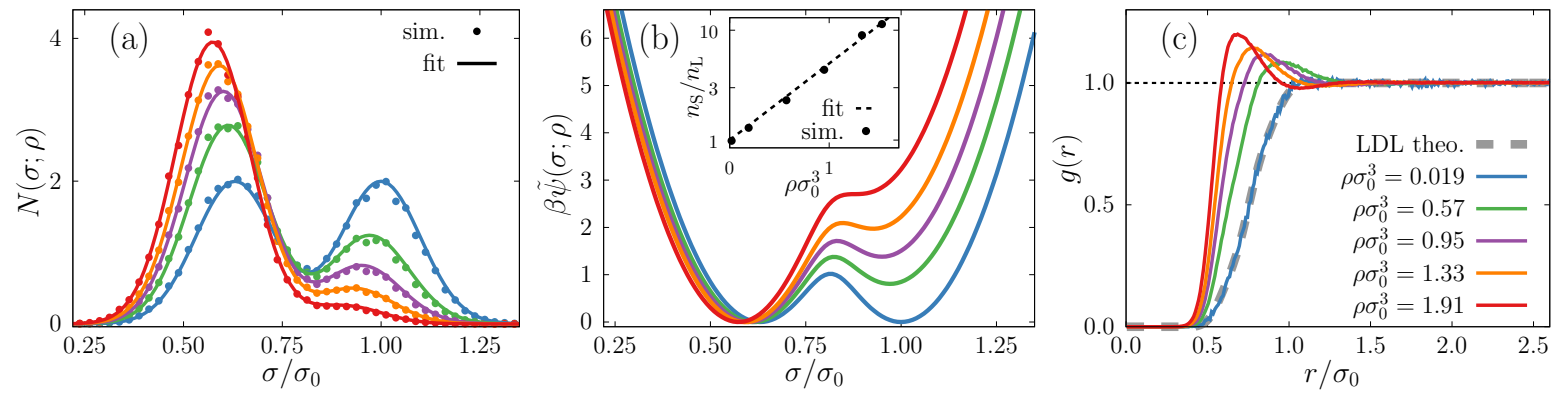

FIG. 2. (a) Emergent size distributions $N(\sigma ; \rho)$, (b) energy landscapes $\tilde{\psi}(\sigma ; \rho)$, and (c) RDF $g(r)$ for varying number densities $\rho$. The distributions in (a) can be well fitted to bimodal distributions of form of Eq. (2) [39, which we take to compute $\tilde{\psi}(\sigma ; \rho)$ in (b) using Eq. (4). The inset in (b) shows the population ratio between small $(\mathrm{S})$ and large $(\mathrm{L})$ states versus $\rho$, calculated from integrations over the distribution, $n_{S} / n_{L}=\int_{-\infty}^{\mathrm{TS}} N(\sigma) \mathrm{d} \sigma / \int_{\mathrm{TS}}^{\infty} N(\sigma) \mathrm{d} \sigma$. The dashed gray line in (c) shows the theoretical estimate in the LDL given by $g(r) \approx \int \mathrm{d} \sigma^{\prime} \int \mathrm{d} \sigma p\left(\sigma^{\prime}\right) p(\sigma) \exp \left[-\beta \phi\left(r ; \sigma, \sigma^{\prime}\right)\right]$.

Resulting emergent size distributions $N(\sigma ; \rho)$ are presented in Fig. 2(a). At dense packing, the particles are compressed and the bimodal distribution shifts to stabilize smaller sizes. For all densities, the $N(\sigma ; \rho)$ distributions also fit well to bimodal Gaussians (Eq. (2)) [39]. Fig. 2(b) shows the crowding-modified landscapes $\beta \tilde{\psi}(\sigma ; \rho)=-\ln N(\sigma ; \rho)$ computed using the fitted $N(\sigma ; \rho)$ distributions. Small sizes are substantially stabilized over the larger states with increasing density, with small-to-large population ratios exceeding a factor of 10, see the inset of Fig. 2(b). This is in agreement with earlier computational studies which reported crowding induced coil-to-globule transitions of two-state biopolymer systems consistent with experiments [5]. Importantly, the transition barriers are drastically modified by packing, which is the key to characterize the changes in the transition kinetics.

For further analysis, we introduce the two-body density-size distribution function [19], $g\left(\mathbf{x}, \mathbf{x}^{\prime}\right) \equiv g\left(r ; \sigma, \sigma^{\prime}\right)$. By integrating out $\sigma$ and $\sigma^{\prime}[19]$, we recover the usual radial distribution function $(\mathrm{RDF}) g(r)$, which represents the average over the polydisperse distributions. Due to the - on average - smaller size of the particles, the first peak of the RDF, shown in Fig. 2(c), shifts to smaller interparticle distances with increasing $\rho$. The first peak grows for higher density as expected from enhanced packing correlations, although the decreasing particle size counterbalances this effect. This can be rationalized by the effective packing fraction, defined as $\eta=\pi \rho\left\langle\sigma^{3}\right\rangle / 6$ [24], which approaches sublinear behavior with increasing $\rho$ [39]. 
Such a weak scaling with density may indeed facilitate homeocrowding as hypothesized for biological cells [10].

From our simulations we directly access the mean first passage times (MFPTs) $\tau^{\mathrm{FP}}$ for small to large (SL) or large to small (LS) transitions from time series analysis [39, 40]. For our overdamped systems, Kramers' escape theory applies [30], which essentially reads

$$
\tau^{\mathrm{FP}}(\alpha, \rho) \sim \alpha^{-1} \exp \left(-\beta E^{a}(\rho)\right)
$$

where $E^{a}(\rho)$ is the energy barrier obtained from $\tilde{\psi}(\sigma ; \rho)$, cf. Fig. $2(\mathrm{~b})$. To check the prefactor scaling with $\alpha$, we plot first the SL-MFPT, $\tau_{\mathrm{SL}}^{\mathrm{FP}}$, as a function of $\alpha$ in Fig. 3(a). Indeed, we observe a clear $\alpha^{-1}$ dependence for all densities, that is, the intrinsic time scale of $\sigma$ simply scales the MFPT linearly.

The density-dependence of the MFPTs, though, is non-trivial and dictated by selfcrowding induced changes to $\tilde{\psi}(\sigma ; \rho)$, and consequently the changes in the activation energies $E^{a}(\rho)$ (see Figs. 11(a,b) and 2(b)). In particular, the SL transition slows because of an increasing energy barrier with density, while the LS transition accelerates. The calculated energy barriers are presented in Fig. 3(b). They depend apparently linearly on the density, changing by up to a factor 2-3, due to the compression of the energy landscapes. This change of activation energy results in a drastic, almost one-order-of magnitude spread of all MFPTs versus density, as presented in Fig. 3(c) as a log-lin plot. We observe that the MFPTs depend exponentially on the crowding density. [We note here that we find, as expected, the exponential relationship between times $\tau^{\mathrm{FP}}$ and energy $E^{a}$ holds across all $\rho$ [39], as predicted by Kramers', Eq. (6).] At a critical density of about $\rho \sigma_{0}^{3} \simeq 1.7$ the transition vanishes in our systems because the L-state becomes unstable, as indicated by the saddle point in the distribution. Here, $\tau_{\mathrm{LS}}^{\mathrm{FP}}$ accordingly approaches zero, which can be interpreted as a completely stabilized (folded or globule) S-state.

The LS-transition can be identified with a folding transition from coil to globule states [7, 8]; qualitatively consistent with our results, it was demonstrated experimentally and through simulations that crowding enhances biomolecular folding rates by a multiple, while unfolding rates decrease [6, 8]. Similarly, large crowders speed up two-state polymer looping kinetics in DNA models [9]. Note, however, that the latter systems dealt with inert, non-responsive crowders while in our homo-crowding case the system responds collective and self-consistent, i.e., the internal kinetics responds in quorum for all particles in the same way. 

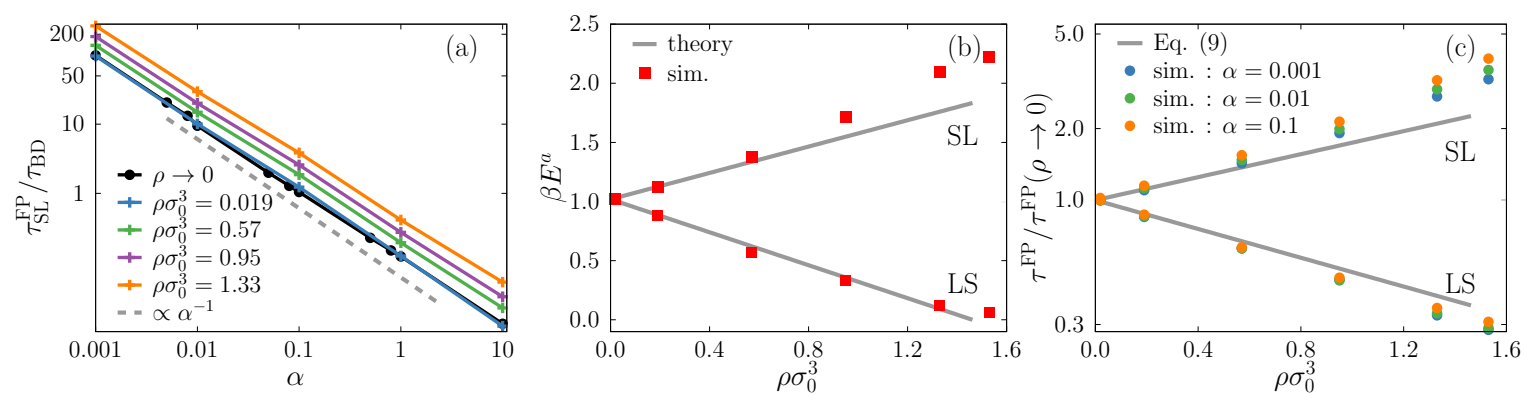

FIG. 3. (a) Simulation MFPTs of small to large (SL) size switching (in units of the Brownian time scale $\left.\tau_{\mathrm{BD}}\right)$ versus $\alpha$ at various densities shown using $\log -\log$ scale. The dashed gray line denotes the $\alpha^{-1}$ Kramers' scaling behavior, Eq. (6). (b) Comparison of theory (lines) and simulations (sim., red squares) of the activation energy $E^{a}$ versus density $\rho$. (c) Comparison of theory and simulations of the MFPT (scaled by the LDL) as a function of $\rho$, using a log-lin scale.

We finally present a perturbation theory, leading to scaling laws which describe the density-dependence of the switching kinetics quantitatively. For this, we consider the mean force on the particle size coordinate due to the interacting environment

$$
F(\sigma)=-\rho \int_{V} \mathrm{~d}^{3} r \int_{-\infty}^{\infty} \mathrm{d} \sigma^{\prime} N\left(\sigma^{\prime}\right) \frac{\partial \phi\left(r ; \sigma, \sigma^{\prime}\right)}{\partial \sigma} g\left(r ; \sigma, \sigma^{\prime}\right) .
$$

This force is the source for the compression and tilt of the energy landscape $\psi$. In a first order perturbation approach to the spatial structure we apply the LDL $g\left(r ; \sigma, \sigma^{\prime}\right) \approx$ $\exp \left[-\beta \phi\left(r ; \sigma, \sigma^{\prime}\right)\right]$. By integrating the force up to the particle's size, $\mathcal{F}(\sigma)=-\int_{0}^{\sigma} \mathrm{d} \sigma^{\prime} F\left(\sigma^{\prime}\right)$, this results in a free energy of the analytical form linear in $\rho$ (with details in [39])

$$
\mathcal{F}(\sigma)=\frac{5}{12} \pi \rho \epsilon \kappa\left(\sigma^{3}+3 \sigma^{2}\langle\sigma\rangle+3 \sigma\left\langle\sigma^{2}\right\rangle\right),
$$

where $\kappa \approx 6.377 \cdot 10^{-4}$ from numerical evaluation for $\beta \epsilon=500$ in the Hertzian pair potential, and $\mathcal{F}(\sigma)$ can be conveniently expressed by the expectation values $\langle\sigma\rangle$ and $\left\langle\sigma^{2}\right\rangle$ with respect to the emergent distribution. From another first order perturbation approach, $N(\sigma ; \rho) \simeq$ $p(\sigma)$ in Eq. (7), we obtain analytically $\langle\sigma\rangle=\left(\mu_{1}+\mu_{2}\right) / 2$ and $\left\langle\sigma^{2}\right\rangle=\delta^{2}+\left(\mu_{1}^{2}+\mu_{2}^{2}\right) / 2$.

Employing the modified free energy landscape $\tilde{\psi}(\sigma) \simeq \psi(\sigma)+\mathcal{F}(\sigma)$ in Kramers' theory, Eq. (6), we obtain following linear forms in $\rho$ for the activation energies, $E_{\mathrm{SL}}^{a} \approx \tilde{\psi}\left(\mu_{3}\right)-$ $\tilde{\psi}\left(\mu_{1}\right)=E_{0}^{a}+\frac{\nu_{\mathrm{SL}}}{\beta} \rho \sigma_{0}^{3}$ and $E_{\mathrm{LS}}^{a} \approx \tilde{\psi}\left(\mu_{3}\right)-\tilde{\psi}\left(\mu_{2}\right)=E_{0}^{a}-\frac{\nu_{\mathrm{LS}}}{\beta} \rho \sigma_{0}^{3}$ with $\beta E_{0}^{a}=1.019$, and $\nu_{\mathrm{SL}}=0.557$ and $\nu_{\mathrm{LS}}=0.697$ from evaluation of Eq. (8) [39]. The theoretical results are shown through lines in Fig. 3(b) and compare very well with the simulations. 
Applying Kramer's rule, $\tau^{\mathrm{FP}} \propto \exp \left(\beta E^{a}\right)$, now leads to MFPT predictions

$$
\tau_{\mathrm{SL}}^{\mathrm{FP}}(\rho) \propto\left(\exp \left(\tilde{\nu}_{\mathrm{SL}}\right)\right)^{\rho} \text { and } \tau_{\mathrm{LS}}^{\mathrm{FP}}(\rho) \propto\left(\exp \left(\tilde{\nu}_{\mathrm{LS}}\right)\right)^{-\rho}
$$

with $\tilde{\nu}_{\mathrm{SL}}=\nu_{\mathrm{SL}} \sigma_{0}^{3} / \beta$ and $\tilde{\nu}_{\mathrm{LS}}=\nu_{\mathrm{LS}} \sigma_{0}^{3} / \beta$, establishing exponential scaling laws for the dependence of the MFPT on the packing density. As with the activation energies, the theoretical predictions for $\tau^{\mathrm{FP}}$ are in very good agreement with the computational results, cf. Fig. 3(c). From the nature of the perturbation theory, the scaling laws are quite universal for any pair potential in the LDL. In our case, however, it works up to surprisingly high densities. The reason lies on one hand in the softness of the Hertzian pair potential, featuring only weakly correlated RDFs, cf. Fig. 2, On the other hand, our parent distribution is relatively broad, perturbing the energy landscape of the internal DOF only linearly in leading order, cf. Eq. (8).

Our work provides a general methodical framework for studying the effects of (homeo)crowding on the internal kinetics in complex responsive systems; this may include multicomponent mixtures, complex (polymodal) energy landscapes, and various internal DOFs. It will be fascinating to add internal activity [41] into the RC model to study actively self-regulated crowding effects. For example, internally fueled switching of hydrogel properties [12, 27, 28] are expected to lead to complex collective nonequilibrium effects [42, 43] and may be programmable to mimic the quorum sensing displayed by bacterial populations [11, 13].

The authors acknowledge support for computing facilities provided by the High Performance Cluster framework by the state of Baden-Württemberg (bwHPC) and the German Research Foundation (DFG) through grant no. INST 39/963-1 FUGG (bwForCluster NEMO).

[1] R. Ellis, Macromolecular crowding: obvious but underappreciated, Trends Biochem. Sci. 26, $597(2001)$.

[2] H.-X. Zhou, G. Rivas, and A. P. Minton, Macromolecular crowding and confinement: Biochemical, biophysical, and potential physiological consequences, Annu. Rev. Biophys. 37, 375 (2008). 
[3] M. Morelli, R. Allen, and P. Rein ten Wolde, Effects of macromolecular crowding on genetic networks, Biophysical Journal 101, 2882 (2011).

[4] Y. C. Kim and J. Mittal, Crowding induced entropy-enthalpy compensation in protein association equilibria, Phys. Rev. Lett. 110, 208102 (2013).

[5] H. Kang, P. A. Pincus, C. Hyeon, and D. Thirumalai, Effects of macromolecular crowding on the collapse of biopolymers, Phys. Rev. Lett. 114, 068303 (2015).

[6] M. S. Cheung, D. Klimov, and D. Thirumalai, Molecular crowding enhances native state stability and refolding rates of globular proteins, Proc. Natl. Acad. Sci. U.S.A. 102, 4753 (2005).

[7] J. Hong and L. M. Gierasch, Macromolecular crowding remodels the energy landscape of a protein by favoring a more compact unfolded state, J. Am. Chem. Soc. 132, 10445 (2010).

[8] N. F. Dupuis, E. D. Holmstrom, and D. J. Nesbitt, Molecular-crowding effects on singlemolecule rna folding/unfolding thermodynamics and kinetics, Proc. Natl. Acad. Sci. U.S.A. 111, $8464(2014)$.

[9] J. Shin, A. G. Cherstvy, and R. Metzler, Kinetics of polymer looping with macromolecular crowding: effects of volume fraction and crowder size, Soft Matter 11, 472 (2015).

[10] J. van den Berg, A. J. Boersma, and B. Poolman, Microorganisms maintain crowding homeostasis, Nat. Rev. Microbiol. 15, 309 (2017).

[11] M. B. Miller and B. L. Bassler, Quorum sensing in bacteria, Annu. Rev. Microbiol. 55, 165 (2001).

[12] T. Heuser, E. Weyandt, and A. Walther, Biocatalytic feedback-driven temporal programming of self-regulating peptide hydrogels, Angew. Chem., Int. Ed. 54, 13258 (2015).

[13] A. Walther, Viewpoint: From responsive to adaptive and interactive materials and materials systems: A roadmap, Adv. Mater. 32, 1905111 (2019).

[14] L. A. Lyon and A. Fernandez-Nieves, The polymer/colloid duality of microgel suspensions, Annu. Rev. Phys. Chem. 63, 25 (2012).

[15] R. Keidel, A. Ghavami, D. M. Lugo, G. Lotze, O. Virtanen, P. Beumers, J. S. Pedersen, A. Bardow, R. G. Winkler, and W. Richtering, Time-resolved structural evolution during the collapse of responsive hydrogels: The microgel-to-particle transition, Sci. Adv. 4, eaao7086 (2018).

[16] M. A. C. Stuart, W. T. S. Huck, J. Genzer, M. Müller, C. Ober, M. Stamm, G. B. Sukhorukov, 
I. Szleifer, V. V. Tsukruk, M. Urban, F. Winnik, S. Zauscher, I. Luzinov, and S. Minko, Emerging applications of stimuli-responsive polymer materials, Nat. Mater. 9, 101 (2010).

[17] J. Mittal and R. B. Best, Dependence of protein folding stability and dynamics on the density and composition of macromolecular crowders, Biophys. J. 98, 315 (2010).

[18] A. R. Denton and M. Schmidt, Colloid-induced polymer compression, J. Phys. Cond. Matt. 14, 12051 (2002).

[19] Y.-C. Lin, B. Rotenberg, and J. Dzubiella, Structure and position-dependent properties of inhomogeneous suspensions of responsive colloids, Phys. Rev. E 102, 042602 (2020).

[20] I. M. Ilie, W. K. den Otter, and W. J. Briels, A coarse grained protein model with internal degrees of freedom. application to $\alpha$-synuclein aggregation, J. Chem. Phys. 144, 085103 (2016).

[21] G. Kapteijns, W. Ji, C. Brito, M. Wyart, and E. Lerner, Fast generation of ultrastable computer glasses by minimization of an augmented potential energy, Phys. Rev. E 99, 012106 (2019).

[22] L. Berthier, E. Flenner, C. J. Fullerton, C. Scalliet, and M. Singh, Efficient swap algorithms for molecular dynamics simulations of equilibrium supercooled liquids, J. Stat. Mech. Theory Exp. 2019, 064004 (2019).

[23] S. Ciarella, M. Rey, J. Harrer, N. Holstein, M. Ickler, H. Löwen, N. Vogel, and L. M. C. Janssen, Soft particles at liquid interfaces: From molecular particle architecture to collective phase behavior, Langmuir 37, 5364 (2021).

[24] U. Baul and J. Dzubiella, Structure and dynamics of responsive colloids with dynamical polydispersity, J. Phys. Condens. Matter 33, 174002 (2021).

[25] U. B. Choi, J. J. McCann, K. R. Weninger, and M. E. Bowen, Beyond the random coil: Stochastic conformational switching in intrinsically disordered proteins, Structure 19, 566 (2011).

[26] S. Dhiman, A. Jain, and S. J. George, Transient helicity: Fuel-driven temporal control over conformational switching in a supramolecular polymer, Angew. Chem., Int. Ed. 56, 1329 (2017).

[27] R. Yoshida, T. Takahashi, T. Yamaguchi, and H. Ichijo, Self-oscillating gel, J. Am. Chem. Soc. 118, 5134 (1996).

[28] L. Heinen, T. Heuser, A. Steinschulte, and A. Walther, Antagonistic enzymes in a biocatalytic ph feedback system program autonomous dna hydrogel life cycles, Nano Lett. 17, 4989 (2017). 
[29] H. Che, S. Cao, and J. C. M. van Hest, Feedback-induced temporal control of breathing polymersomes to create self-adaptive nanoreactors, J. Am. Chem. Soc. 140, 5356 (2018).

[30] P. Hänggi, P. Talkner, and M. Borkovec, Reaction-rate theory: fifty years after kramers, Rev. Mod. Phys. 62, 251 (1990).

[31] J.-P. Hansen and I. R. McDonald, Theory of Simple Liquids, 4th ed. (Academic Press, Oxford, 2013).

[32] L. Rovigatti, N. Gnan, A. Ninarello, and E. Zaccarelli, Connecting elasticity and effective interactions of neutral microgels: the validity of the hertzian model, Macromolecules 52, 4895 (2019).

[33] M. Urich and A. R. Denton, Swelling, structure, and phase stability of compressible microgels, Soft Matter 12, 9086 (2016).

[34] M. J. Bergman, N. Gnan, M. Obiols-Rabasa, J.-M. Meijer, L. Rovigatti, E. Zaccarelli, and P. Schurtenberger, A new look at effective interactions between microgel particles, Nat. Commun. 9, 5039 (2018).

[35] P. S. Mohanty, D. Paloli, J. J. Crassous, E. Zaccarelli, and P. Schurtenberger, Effective interactions between soft-repulsive colloids: Experiments, theory, and simulations, J. Chem. Phys. 140, 094901 (2014)

[36] H. J. C. Berendsen, Simulating the Physical World (Cambridge University Press, Cambridge, 2007).

[37] M. P. Allen and D. J. Tildesley, Computer Simulations of Liquids, 2nd ed. (Oxford University Press, Oxford, 2017).

[38] E. Yamamoto, T. Akimoto, A. Mitsutake, and R. Metzler, Universal relation between instantaneous diffusivity and radius of gyration of proteins in aqueous solution, Phys. Rev. Lett. 126, $128101(2021)$

[39] See the Supplemental Material at http://link.aps.org/ supplemental/xxx for details on the simulation parameters, perturbation theory, fit parameters for emergent bimodal distributions, calculation of mean first passage times, and verification of the Arrhenius behavior in the simulations.

[40] M. Hinczewski, Y. von Hansen, J. Dzubiella, and R. R. Netz, How the diffusivity profile reduces the arbitrariness of protein folding free energies, J. Chem. Phys. 132, 245103 (2010).

[41] C. Bechinger, R. Di Leonardo, H. Löwen, C. Reichhardt, G. Volpe, and G. Volpe, Active 
particles in complex and crowded environments, Rev. Mod. Phys. 88, 045006 (2016).

[42] A. Moncho-Jordá and J. Dzubiella, Controlling the microstructure and phase behavior of confined soft colloids by active interaction switching, Phys. Rev. Lett. 125, 078001 (2020).

[43] M. Bley, A. Moncho-Jordá, and J. Dzubiella, Active binary switching of soft colloids: stability and structural properties, Soft Matter 17, xxxx (2021). 